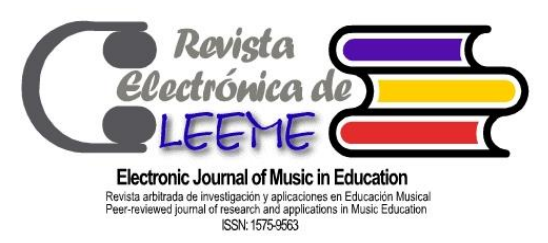

\title{
La formación en TIC de los pedagogos de música. Análisis de la situación en las Enseñanzas Superiores de Música
}

\author{
The ICT training of music pedagogues. Analysis of the situation in postsecondary \\ studies
}

\author{
Nuria Díez Latorre \\ ndiezlatorre@gmail.es \\ Facultad de Educación, Psicología y Trabajo Social \\ Universidad de Lérida \\ Lérida, España \\ ORCID: https://orcid.org/0000-0002-7421-9289
}

doi: 10.7203/LEEME.42.13067

Recibido: 20-09-2018 Aceptado: 17-10-2018. Contacto y correspondencia: Nuria Díez Latorre, Facultad de Educación, Psicología y Trabajo Social Universidad de Lérida, Facultad de Educación, Psicología y Trabajo Social, Avda. del Estudio General, 4, 25001 Lérida. España.

\section{Resumen}

La incorporación de las tecnologías de la información y la comunicación (TIC) a la enseñanza en los conservatorios de música es, a día de hoy, muy escasa y desigual. Independientemente de otros factores, uno de los motivos puede ser la falta de formación del profesorado en esta materia; sin embargo, sobre este tema existe todavía muy poca información. Por ello, este informe ofrece una panorámica sobre la formación en TIC que reciben actualmente los futuros pedagogos de música durante sus estudios en el conservatorio superior. Para ello se ha llevado a cabo un extenso análisis de contenido de los planes de estudio de Pedagogía Musical y guías docentes de catorce centros repartidos por todo el territorio español. Los resultados revelan que, en general, los contenidos tecnológicos se centran en el manejo de software musical pero la formación en TIC para la educación es bastante deficitaria y existen grandes diferencias entre los distintos centros.

Palabras clave: Tecnologías de la información y la comunicación (TIC), formación inicial docente, pedagogía musical, conservatorio.

\begin{abstract}
Nowadays, the incorporation of information and communication technologies (ICT) into the teaching methods at music conservatories is limited and unequal. Regardless of other factors, one of the reasons may be the lack of teacher training in this area; however, there is still very little information on this subject. Therefore, this report offers an overview of the ICT training currently offered to future music pedagogues during their studies at postsecondary Conservatories. For this purpose, an extensive content analysis of the Music Pedagogy curricula and teaching guides of fourteen centers spread throughout the Spanish territory has been carried out. The results reveal that, in general, the technological contents are focused on the management of musical software but ICT training for education is quite deficient and there are great differences among the centers.
\end{abstract}

Key words: Communication and information technologies (ICT), initial teacher training, musical pedagogy, conservatory. 


\section{Introducción}

La enseñanza en los conservatorios españoles presenta una cierta resistencia a la innovación pedagógica con las TIC que sí se está desarrollando en otros contextos educativos. En estos centros, se forma al alumnado en diversas destrezas musicales, además del estudio individual de un instrumento. A excepción de esto último, el resto de asignaturas son colectivas, como la de Lenguaje Musical, que se imparte durante seis cursos. Ésta se considera una de las materias más importantes del plan de estudios porque aglutina toda la base de contenidos teóricoprácticos, habilidades y aptitudes que el alumnado necesita para su formación integral como músicos. Sin embargo, resulta difícil para muchos de ellos que, además, perciben esta asignatura menos atractiva y motivadora que otras (Ordoñana, Laucirica y Tejada, 2004; Berrón, Balsera y Monreal, 2017). Sería muy interesante aprovechar las ventajas que ofrecen las nuevas tecnologías en la docencia de esta y otras materias. Diferentes investigaciones reflejan resultados positivos en varios aspectos del aprendizaje gracias al uso de software musical, tales como una mejora en la lectura entonada (Galera, Tejada y Trigo, 2013), en el desarrollo del oído (Balo, Lago y Ponce de León, 2014) y en la mejora de destrezas rítmicas (Ordoñana, Laucirica y Tejada, 2004; Tejada, 2010). Otros estudios han comprobado los beneficios del uso de la pizarra digital en el aula (Bernabé y Azorín, 2015), de la utilización de recursos de Internet para enriquecer el aprendizaje presencial y creación de blogs participativos donde el alumnado comparte conocimientos (Martínez, 2017).

Sin embargo, como hemos adelantado, en los conservatorios no existe una normalización en el uso de las TIC y su integración es lenta y dispar. Un factor que puede estar influyendo en ello es la falta de formación en tecnologías y en su uso didáctico por parte del profesorado. Es, precisamente, en la formación inicial de los docentes donde creemos que debería comenzar la implementación de las TIC. Durante este período, el futuro pedagogo adquiere no sólo una suma de habilidades y conocimientos, sino que ha de desarrollar una actitud reflexiva sobre la enseñanza y sobre las implicaciones de su profesión en la que las tecnologías deben tener cabida. Dado que estos docentes reciben su formación en el conservatorio superior sin necesidad de realizar otros estudios universitarios, resulta muy conveniente que las TIC se tengan en cuenta a la hora de diseñar los planes de estudio y los contenidos de las asignaturas. Por tanto, el propósito de esta investigación es responder a la siguiente pregunta: ¿cuál es la situación actual de la formación en TIC que recibe el alumnado de Pedagogía Musical en los conservatorios superiores españoles?

\section{Revisión teórica}

En un contexto en el que las TIC ganan cada vez más presencia, resulta imprescindible que el ámbito educativo se haga eco de los cambios, de los retos y de las necesidades que demanda la sociedad actual. La formación del profesorado constituye un factor influyente en los procesos de cambio e innovación educativa. Para Sancho, Bosco, Alonso y Sánchez (2015), se trata de un proceso continuo que debe proporcionar al docente:

@NURIA DÍEZ LATORRE. THE CONTENT OF THIS ARTICLE IS THE SOLE RESPONSIBILITY OF THE AUTHORS. THE REVISTA ELECTRÓNICA DE LEEME AND UNIVERSITAT DE VALĖNCIA ARE NOT LIABLE FOR ANY LEGAL ACTIONS THAT MAY ARISE INVOLVING THE ARTICLE'S CONTENT. REVISTA ELECTRÓNICA DE LEEME -LISTA ELECTRÓNICA EUROPEA DE MÚSICA EN LA EDUCACIÓN-HTTP://OJS.UV.ES/INDEX.PHP/LEEME/INDEX ISSN: 1575-9563 EDITORES: UNIVERSIDAD DE VALENCIA Y JESÚS TEJADA GIMÉNEZ. VISIBILIDAD DE ESTA REVISTA: SCOPUS, EMERGING SOURCES CITATION INDEX (CLARIVATE), EBSCO, CINDOC (CSIC), CITEFACTOR, COPAC, DIALNET, DICE (CSIC), DOAJ, E-REVISTAS (CSIC), EBSCO PREMIER, ERIH+,

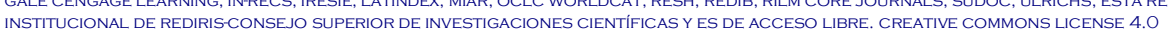


- Entendimiento sobre las dimensiones de su labor en la sociedad.

- Desarrollo de habilidades e intereses que permitan establecer relaciones de aprendizaje productivas con el alumnado.

- Capacidad para pensar formas alternativas a la enseñanza tradicional.

- Criterios para analizar las consecuencias de los cambios producidos por las TIC en el aprendizaje y en la creación, representación, almacenamiento y acceso al conocimiento.

- Planteamiento crítico sobre el papel de los recursos digitales en la enseñanza, por un lado; y en la vida y forma de aprender y relacionarse de los estudiantes, por otro.

Los dos últimos puntos tienen relación con las voces críticas -que también se deben tomar en consideración- acerca del uso de las TIC. Moreno (2018) recoge diversas desventajas tales como la posibilidad de distracción y de empeoramiento de la comunicación directa, la despersonalización de la enseñanza, el uso de fuentes de información de baja calidad o la dificultad de acceso a dispositivos electrónicos por parte del alumnado, entre otras.

La formación inicial, no obstante, es el momento idóneo para predisponer a los docentes hacia una integración reflexiva de las TIC en su práctica educativa (Gutiérrez, Palacios y Torrego, 2010). El profesorado precisa el desarrollo de conocimientos y competencias con respecto a las TIC ya desde su formación inicial, que debe abarcar distintas áreas. Sin embargo, se considera que esta formación es instrumentalista e insuficiente, por lo que se reclama una formación más pedagógica para el uso de las TIC en el proceso de enseñanza-aprendizaje (Losada, Valverde y Correa, 2012; Fernández, Fernández y Cebreiro, 2016).

Paredes, Guitert y Rubia (2015) afirman que, aunque la formación practicada por las distintas universidades aplica ciertos principios que intentan generar transformaciones, la innovación, el cambio y la renovación no han sido objeto de esta formación inicial. Esto puede explicar que, en las escuelas, las prácticas educativas que incluyen las TIC no se alejan demasiado de las prácticas tradicionales, pero con cierto matiz tecnológico. Con respecto a esta idea, por un lado, Coll (2009) sostiene que se debería aprovechar la potencialidad de las tecnologías para impulsar nuevas formas de enseñanza y aprendizaje; y, por otro lado, Silva y Astudillo (2012) señalan la existencia de diferentes barreras que complican la integración de la tecnología en la formación de los futuros docentes:

- En las facultades, no existe una visión clara de cómo integrar las TIC en las propias prácticas pedagógicas del profesorado. Se tiende a utilizar el modelo instruccional tradicional, lo que dificulta que el alumnado pueda integrar las TIC en sus futuras clases como docentes.

- Se depende de una sola asignatura de tecnología, lo que provoca una visión separada del resto de materias formativas relacionadas con la pedagogía y la didáctica. Se hace necesario, además, una visión más transversal de las TIC a lo largo de todo el plan de estudios.

- La falta de habilidad del discente para transferir sus habilidades con las TIC al aula e integrarlas en las prácticas docentes, al margen de su alfabetización en el uso técnico de las mismas. @NURIA DIEZZ LATORRE. THE CONTENT OF THIS ARTICLE IS THE SOLE RESPONSIBIIITY OF THE AUTHORS. THE REVISTA ELECTRÓNICA DE LEEME AND UNIVERSITAT DE VALĖNCIA ARE
NOT LIABLE FOR ANY LEGAL ACTIONS THAT MAY ARISE INVOLVING THE ARTICLE'S CONTENT. REVISTA ELECTRÓNICA DE LEEME -LISTA ELECTRÓNICA EUROPEA DE MÚSICA EN LA EDUCACIÓN-HTTP://OJS.UV.ES/INDEX.PHP/LEEME/INDEX ISSN: 1575-9563 EDITORES: UNIVERSIDAD DE VALENCIA Y JESÚS TEJADA GIMÉNEZ. VISIBILIDAD DE ESTA REVISTA: SCOPUS, EMERGING SOURCES CITATION INDEX (CLARIVATE), EBSCO, CINDOC (CSIC), CITEFACTOR, COPAC, DIALNET, DICE (CSIC), DOAJ, E-REVISTAS (CSIC), EBSCO PREMIER, ERIH+, GALE CENGAGE LEARNING, IN-RECS, IRESIE, LATINDEX, MIAR, OCLC WORLDCAT, RESH, REDIB, RILM CORE JOURNALS, SUDOC, ULRICHS, ESTA RE
INSTITUCIONAL DE REDIRIS-CONSEJO SUPERIOR DE INVESTIGACIONES CIENTIFICAS Y ES DE ACCESO LIBRE. CREATIVE COMMONS LICENSE 4.0 
En el ámbito de las enseñanzas artísticas de música, esta formación inicial docente se adquiere en los conservatorios superiores. El Real Decreto 631/2010, que regula el contenido básico de cada especialidad, fija un mínimo de 6 créditos para la materia "Tecnología Musical" en el caso de la especialidad de Pedagogía Musical, además de una serie de contenidos de carácter general. Corresponde a cada administración elaborar un plan de estudios propio que defina las asignaturas, desarrolle los contenidos e, incluso, incremente el número de créditos de ésta y otras materias. Sobre los contenidos y las características de la formación tecnológica que brindan los conservatorios superiores hay poca literatura científica hasta la fecha. Las aportaciones provienen más bien de trabajos sobre la formación de los docentes de educación musical en la Enseñanza Secundaria, que, en su mayoría, ha sido también formados en un conservatorio superior (Colás y Hernández, 2014), así como de las conclusiones de investigaciones con otros objetivos pero que también dedican unas palabras al respecto (Belló y González, 2010; De la Rosa, 2015; Timiraos, 2015). De estos estudios se desprende que, a pesar de mostrar un interés positivo hacia las tecnologías, en general, la preparación del profesorado en TIC es deficitaria. Este factor impide que tengan lugar cambios sustanciales en la práctica pedagógica, puesto que los docentes no se sienten preparados ni seguros para integrar las TIC más allá de una cierta actualización para hacer más efectiva la metodología tradicional.

\section{Método}

\subsection{Diseño}

Para llevar a cabo esta investigación, se ha realizado un análisis documental basado en los planes de estudio y las guías docentes del curso 2017-2018 de los conservatorios superiores españoles que imparten la especialidad de Pedagogía Musical. En este sentido, presenta similitud con otros estudios realizados en el ámbito universitario (Losada, Valverde y Correa, 2012; Ballesta y Céspedes, 2015).

Además de valorar de manera externa la cantidad de asignaturas y de créditos estrechamente relacionados con las TIC, se ha optado por indagar el tipo de contenidos y la presencia de las tecnologías en otros aspectos de la enseñanza según se recogen en las guías docentes. Para esto último, siguiendo a Bardin (1996), es preciso delimitar unidades de registro, de contexto y reglas de enumeración para extraer los datos de interés. En el caso de las asignaturas relacionadas directamente con las tecnologías, se han tomado como unidades de registro las frases que especifican los distintos bloques de contenidos. Posteriormente, se han elaborado categorías para su clasificación. En cambio, para el resto de asignaturas se ha optado generalmente por localizar palabras del campo semántico de las tecnologías. Éstas últimas se relacionan con sus respectivas unidades de contexto que son los distintos elementos de las guías docentes: contenidos, objetivos, metodología, evaluación y otros. Finalmente, la enumeración se ha llevado a cabo de dos maneras, según los casos: mediante recuento de frecuencias y determinando la presencia -o no- de referencias a las TIC en las guías docentes. @NURIA DIEZZ LATORRE. THE CONTENT OF THIS ARTICLE IS THE SOLE RESPONSIBILITY OF THE AUTHORS. THE REVISTA ELECTRÓNICA DE LEEME AND UNIVERSITAT DE VALĖNCIA ARE
NOT LIABLE FOR ANY LEGAL ACTIONS THAT MAY ARISE INVOLVING THE ARTICLE'S CONTENT. REVISTA ELECTRÓNICA DE LEEME -LISTA ELECTRÓNICA EUROPEA DE MÚSICA EN LA EDUCACIÓN-HTTP://OJS.UV.ES/INDEX.PHP/LEEME/INDEX ISSN: 1575-9563 EDITORES: UNIVERSIDAD DE VALENCIA Y JESÚS TEJADA GIMEENEZ. VISIBILIDAD DE ESTA REVISTA: SCOPUS, EMERGING SOURCES CITATION INDEX (CLARIVATE), EBSCO, CINDOC (CSIC), CITEFACTOR, COPAC, DIALNET, DICE (CSIC), DOAJ, E-REVISTAS (CSIC), EBSCO PREMIER, ERIH+, INSTITUCIONAL DE REDIRIS-CONSEJO SUPERIOR DE INVESTIGACIONES CIENTIFICAS Y ES DE ACCESO LIBRE. CREATIVE COMMONS LICENSE 4.0 


\subsection{Muestra}

Debido a que no todos los conservatorios superiores ofertan la especialidad, en la investigación se considera un total de 14 centros repartidos en 11 comunidades autónomas (Tabla 1). Así pues, contamos con 11 planes de estudio distintos.

En cuanto a las guías docentes, además de las asignaturas vinculadas explícitamente a la tecnología, se han incluido en la investigación aquellas relacionadas con materias educativas. Cabe decir que no se ha podido contar con todas las guías, dado que no están publicadas o su acceso es restringido. Por otro lado, se han descartado algunas asignaturas que, pese a estar asociadas a estas materias, no tienen relación con nuestro estudio por la naturaleza de sus contenidos. El total de guías analizadas asciende a 102:

Tabla 1. Relación de Administraciones y centros respectivos incluidos en el estudio

\begin{tabular}{cl}
\hline ADMINISTRAIÓN & \multicolumn{1}{c}{ CENTRO } \\
\hline Andalucía & Real Conservatorio de Música de Granada \\
Asturias & Conservatorio Superior de Música del Principado de Asturias \\
Baleares & Conservatorio Superior de Música de las Islas Baleares \\
Canarias & Conservatorio Superior de Música de Canarias \\
Cataluña & Escola Superior de Música de Catalunya \\
C. Valenciana & Conservatorio Superior de Música de Alicante \\
& Conservatorio Superior de Música de Castellón \\
Galicia & Conservatorio Superior de Música de Valencia \\
& Conservatorio Superior de Música de A Coruña \\
Madrid & Conservatorio Superior de Música de Vigo \\
Murcia & Real Conservatorio Superior de Música de Madrid \\
Navarra & Conservatorio Superior de Música de Murcia \\
País Vasco & Conservatorio Superior de Música de Navarra \\
& Musikene, Centro Superior de Música del País Vasco
\end{tabular}

Fuente: Elaboración propia

\subsection{Dimensiones de estudio}

En esta investigación, se plantean dos dimensiones de estudio: (1) el peso de las TIC en los planes de estudio y (2) la integración de las TIC en las guías docentes.

Para la primera, se han considerado dos aspectos o subdimensiones: (a) la distribución en créditos ETCS y por cursos de asignaturas vinculadas a la materia "Tecnología Musical”, y (b) los contenidos detallados en los descriptores de dichas asignaturas y de otras asociadas a materias educativas: "Fundamentos de Pedagogía", "Didáctica de la Educación Musical" y "Organización Educativa".

Para la segunda, se ha procedido de forma distinta en función de la naturaleza de la asignatura. En cuanto a las que están directamente relacionadas con la materia "Tecnología Musical", se han examinado sus contenidos específicos en detalle; sin embargo, se han obviado otros elementos como los objetivos, las competencias, la metodología o la evaluación al considerarlos implícitos en las asignaturas. Por otro lado, con respecto a aquellas relacionadas con materias educativas, se han localizado contenidos y otros elementos curriculares relacionados

@NURIA DIEZZ LATORRE. THE CONTENT OF THIS ARTICLE IS THE SOLE RESPONSIBILITY OF THE AUTHORS. THE REVISTA ELECTRÓNICA DE LEEME AND UNIVERSITAT DE VALĖNCIA ARE NOT LIABLE FOR ANY LEGAL ACTONS THAT MAY ARISE INVOLVING THE ARTICLE'S CONTENT. REVISTA ELECTRÓNICA DE LEEME - USTA ELECTRÓNICA EUROPEA DE MÚSICA EN LA EDUCACIÓN-HTTP://OJS.UV.ES/INDEX.PHP/LEEME/INDEX ISSN: 1575-9563 EDITORES: UNIVERSIDAD DE VALENCIA Y JESÚS TEJADA GIMÉNEZ. VISIBILIDAD DE ESTA REVISTA: SCOPUS, EMERGING SOURCES CITATION INDEX (CLARIVATE), EBSCO, CINDOC (CSIC), CITEFACTOR, COPAC, DIALNET, DICE (CSIC), DOAJ, E-REVISTAS (CSIC), EBSCO PREMIER, ERIH+, GALE CENGAGE LEARNING, IN-RECS, IRESIE, LATINDEX, MIAR, OCLC WORLDCAT, RESH, REDIB, RILM CORE JOURNALS, SUDOC, ULRICHS, ESTA REVISTA ESTÁ PUBLICADA CON EL APOYO INSTITUCIONAL DE REDIRIS-CONSEJO SUPERIOR DE INVESTIGACIONES CIENTIFICAS Y ES DE ACCESO LIBRE. CREATIVE COMMONS LICENSE 4.0 
con la integración de las TIC, como (a) competencias transversales, generales y de la especialidad, (b) resultados de aprendizaje, (c) objetivos, (d) contenidos, (e) metodología y (f) evaluación, que comprende criterios de evaluación, procedimientos, instrumentos y criterios de calificación.

\subsection{Procedimiento}

En relación con la materia "Tecnología Musical”, se ha realizado un examen de los planes de estudio para extraer información relacionada con el número de créditos, asignaturas y cursos. Dicha información se ha registrado mediante una tabla de elaboración propia que se muestra en el apartado de resultados. En cuanto al contenido específico de las asignaturas, se han leído atentamente los descriptores para localizar elementos comunes, distribuirlos en categorías y contabilizar las frecuencias de cada categoría. Posteriormente, en las guías docentes, se ha examinado en detalle el desarrollo de dichos contenidos.

En cuanto a las asignaturas de las otras materias citadas, además de localizar epígrafes relacionados con las TIC en los descriptores de los planes de estudio, se ha realizado una lectura completa de las guías docentes en busca de palabras clave como "tecnología", "tecnológico", "TIC", "software", "digital", "virtual", "web", "Internet", "informática", "multimedia" o "telemático", entre otras. Las alusiones se han señalado en una tabla similar a la de la Figura 1, aunque en los resultados no se muestra esta tabla debido a su extensión. Por otro lado, se han valorado estos elementos a nivel cualitativo.

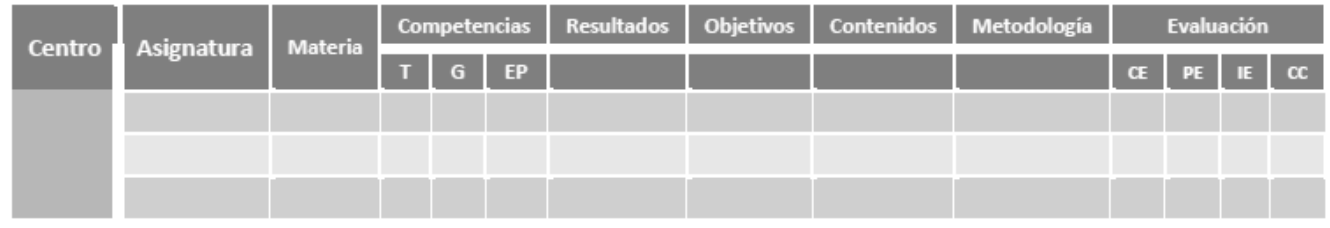

Figura 1. Muestra de tabla para el análisis de las guías docentes

\section{Resultados}

\subsection{Análisis de los planes de estudio}

La Tabla 2 muestra las asignaturas relacionadas con "Tecnología Musical” junto con su distribución de créditos, cursos y/o cuatrimestres. Los datos señalados con un asterisco (*) indican que están incluidas aquellas asignaturas que carecen de relevancia en cuanto a sus contenidos. Por otro lado, las asignaturas marcadas en cursiva se centran en la didáctica con las TIC.

Tabla 2. Distribución de créditos, asignaturas, cursos y cuatrimestres de las asignaturas de Tecnología Musical

\begin{tabular}{|c|c|c|c|c|c|}
\hline & \multirow[t]{2}{*}{ ASIGNATURAS } & \multirow[t]{2}{*}{ ECTS } & \multicolumn{2}{|c|}{ DISTRIBUCIÓN ECTS } & \multirow[t]{2}{*}{ TOTAL } \\
\hline & & & CURSC & IMESTRE & \\
\hline \multirow[t]{2}{*}{ Andalucía* } & $\begin{array}{l}\text { Tecnología informática aplicada a la } \\
\text { música }\end{array}$ & 3 & $1^{\circ}$ & (3) & $7 *$ \\
\hline & Organología y acústica* & $4 *$ & $3^{\circ}$ & (4) & \\
\hline Asturias & Tecnologías aplicadas a la música & 6 & $1^{\mathrm{o}}$ & (6) & 6 \\
\hline
\end{tabular}

@NURIA DIEZ LATORRE. THE CONTENT OF THIS ARTICLE IS THE SOLE RESPONSIBILITY OF THE AUTHORS. THE REVISTA ELECTRÓNICA DE LEEME AND UNIVERSITAT DE VALĖNCIA ARE NOT LIABLE FOR ANY LEGAL ACTIONS THAT MAY ARISE INVOLVING THE ARTICLE'S CONTENT. REVISTA ELECTRÓNICA DE LEEME -LISTA ELECTRÓNICA EUROPEA DE MÚSICA EN LA EDUCACIÓN-HTTP://OJS.UVES/INDEXPHP/LEEME/INDEX ISSN: 1575-9563 EDITORES: UNIVERSIDAD DE VALENCIA Y JESÚS TEJADA GIMÉNEZ. VISIBILIDAD DE ESTA REVISTA: SCOPUS, EMERGING SOURCES CITATION INDEX (CLARIVATE), EBSCO, CINDOC (CSIC), CITEFACTOR, COPAC, DIALNET, DICE (CSIC), DOAJ, E-REVISTAS (CSIC), EBSCO PREMIER, ERIH+, GALE CENGAGE LEARNING, IN-RECS, IRESIE, LATINDEX, MIAR, OCLC WORLDCAT, RESH, REDIB, RILM CORE JOURNALS, SUDOC, ULRICHS, ESTA REVISTA ESTÁ PUBLICADA CON EL APOYO INSTITUCIONAL DE REDIRIS-CONSEJO SUPERIOR DE INVESTIGACIONES CIENTIFICAS Y ES DE ACCESO LIBRE. CREATIVE COMMONS LICENSE 4.0 


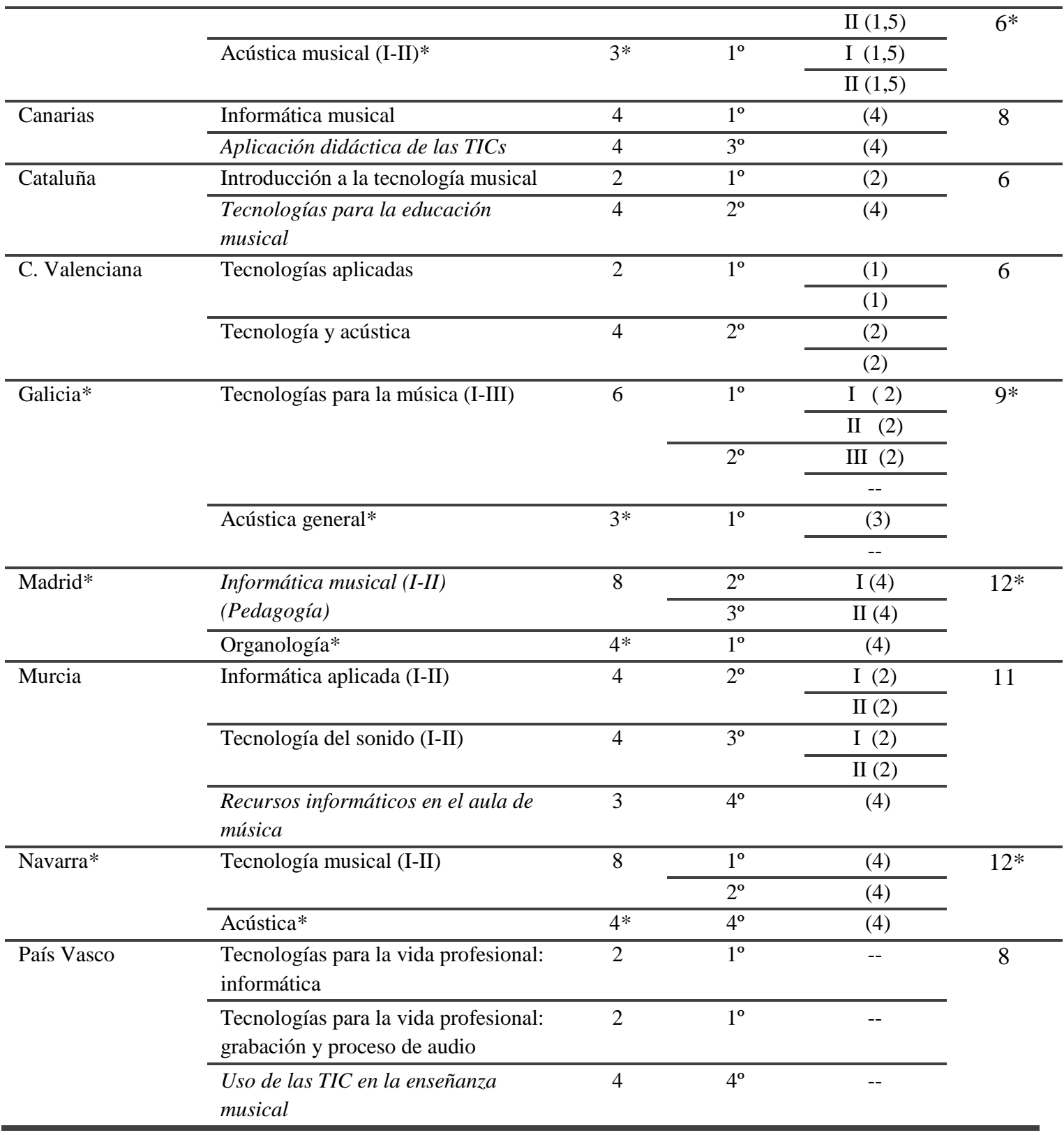

Fuente: Elaboración propia

La Figura 2 presenta los resultados del total de créditos teniendo en cuenta todas las asignaturas y muestra una comparativa entre los planes de estudio. Como puede observarse, los resultados oscilan desde los 6 créditos mínimos estipulados en la norma estatal hasta los 12 alcanzados en Navarra y Madrid. La fracción representativa de los créditos asignados a las TIC en función del total de comunidades se muestra en la Figura 3.

@NURIA DÍEZ LATORRE. THE CONTENT OF THIS ARTICLE IS THE SOLE RESPONSIBILITY OF THE AUTHORS. THE REVISTA ELECTRÓNICA DE LEEME AND UNIVERSITAT DE VALĖNCIA ARE NOT LIABLE FOR ANY LEGAL ACTIONS THAT MAY ARISE INVOLVING THE ARTICLE'S CONTENT. REVISTA ELECTRÓNICA DE LEEME -LISTA ELECTRÓNICA EUROPEA DE MÚSICA EN LA EDUCACIÓN-HTTP://OJS.UV.ES/INDEX.PHP/LEEME/INDEX ISSN: 1575-9563 EDITORES: UNIVERSIDAD DE VALENCIA Y JESÚS TEJADA GIMÉNEZ. VISIBILIDAD DE ESTA REVISTA: SCOPUS, EMERGING SOURCES CITATION INDEX (CLARIVATE), EBSCO, CINDOC (CSIC), CITEFACTOR, COPAC, DIALNET, DICE (CSIC), DOAJ, E-REVISTAS (CSIC), EBSCO PREMIER, ERIH+, INSTITUCIONAL DE REDIRIS-CONSEJO SUPERIOR DE INVESTIGACIONES CIENTIFICAS Y ES DE ACCESO LIBRE. CREATIVE COMMONS LICENSE 4.O 


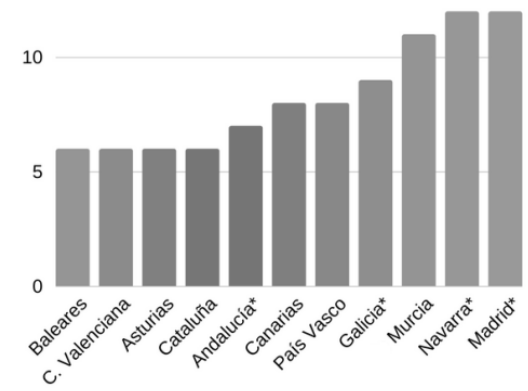

Figura 2. Créditos por plan de estudios

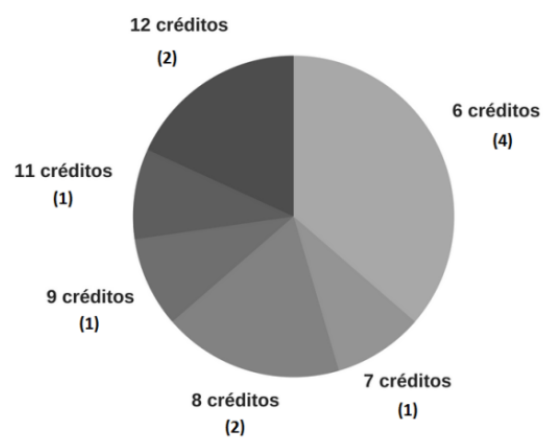

Figura 3. Relación del número de créditos en el conjunto

Por otro lado, la Figura 4 representa el total de créditos sin aquellas asignaturas señaladas con el asterisco -"Acústica" y "Organología”-. En el caso de las comunidades de Andalucía y Baleares, los créditos específicamente dedicados a las TIC se reducen a 3 y también ven disminuida su carga las comunidades de Galicia, Navarra y Madrid. La comunidad que más dedica a las TIC en la formación de sus pedagogos es la Región de Murcia con 11 créditos. La mayoría de planes dedica entre 6 y 8 créditos reales a las tecnologías, como se observa en la Figura 5, lo que supone entre el 2,5\% y el 3,33\% con respecto a los 240 créditos de la titulación. No obstante, si se considera también el mínimo y el máximo, este porcentaje se sitúa entre el $1,25 \%$ y el $4,58 \%$.

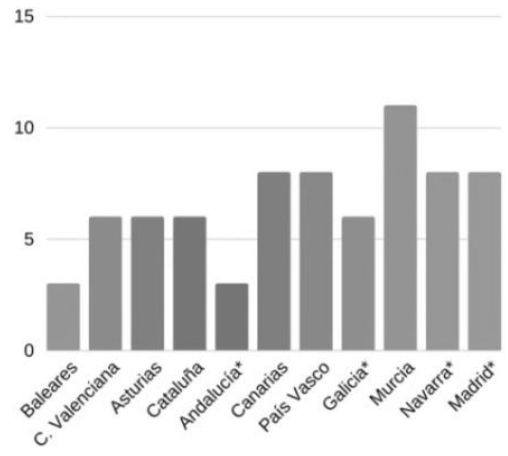

Figura 4. Créditos sin Acústica y Organología

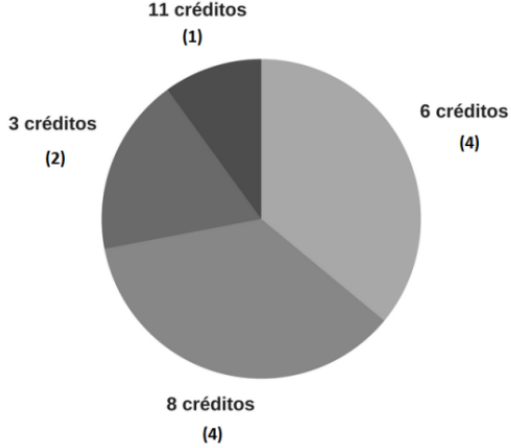

Figura 5. Relación de número de créditos en el conjunto sin Acústica y Organología

En cuanto a la presencia de asignaturas específicas o con una carga importante de TIC para la educación, sólo 5 planes de estudio incluyen alguna, lo que representa un 45\%, (Figura 6). Además, se da la coincidencia de que se destinan 4 créditos a esas asignaturas, excepto en Madrid, que son el doble; en este caso, se trata de una asignatura única que aglutina los contenidos que en otros planes se distribuyen en asignaturas diferentes. Así pues, los créditos dedicados a la formación didáctica con las TIC varían entre $0 \%$ y 3,33\% con respecto al total de 240 de la carrera.

@NURIA DÍEZ LATORRE. THE CONTENT OF THIS ARTICLE IS THE SOLE RESPONSIBILITY OF THE AUTHORS. THE REVISTA ELECTRÓNICA DE LEEME AND UNIVERSITAT DE VALÈNCIA ARE NOT LIABLE FOR ANY LEGAL ACTIONS THAT MAY ARISE INVOLVING THE ARTICLE'S CONTENT. REVISTA ELECTRÓNICA DE LEEME -LISTA ELECTRÓNICA EUROPEA DE MÚSICA EN LA EDUCACIÓN-HTTP://OJS.UV.ES/INDEX.PHP/LEEME/INDEX ISSN: $1575-9563$ EDITORES: UNIVERSIDAD DE VALENCIA Y JESÚS TEJADA GIMÉNEZ. VISIBILIDAD DE ESTA REVISTA: GALE CENGAGE LEARNING, IN-RECS, IRESIE, LATINDEX, MIAR, OCLC WORDCAT, RESH, REDIB, RILM CORE JOURNALS, SUDOC, ULRICHS, ESTA REVISTA ESTA PUBLICADA CON EL APOYO GALE CENGAGE LEARNING, IN-RECS, IRESIE, LATINDEX, MIAR, OCLC WORLDCAT, RESH, REDIB, RILM CORE JOURNALS, SUDOC, ULRICHS, ESTA RE
INSTITUCIONAL DE REDIRIS-CONSEJO SUPERIOR DE INVESTIGACIONES CIENTIFICAS Y ES DE ACCESO LIBRE. CREATIVE COMMONS LICENSE 4.0 
Finalmente, la Figura 7 ilustra la distribución de las asignaturas de "Tecnología Musical" por cursos. Se han ordenado de arriba a abajo en función del curso de inicio: en la parte superior figuran aquellos planes que comienzan antes esta materia y, en la inferior, los que lo hacen más tarde. La mayoría de planes inician la formación tecnológica durante el primer año de la titulación, a excepción de Madrid y la Región de Murcia, que comienzan en el segundo. No obstante, aquellas asignaturas relacionadas con la didáctica se inician más tarde en todos los casos, pero en distinto curso según la comunidad.

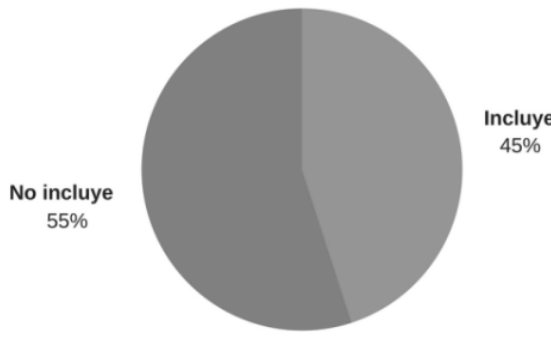

Figura 6. Planes que incluyen asignatura de TIC para la educación

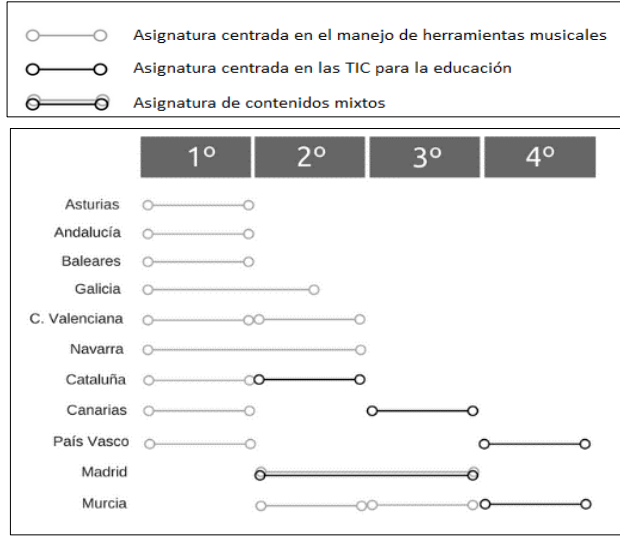

Figura 7. Distribución de asignaturas tecnológicas por cursos

Por otra parte, la Tabla 3 presenta los contenidos de estas asignaturas. Se han elaborado 7 categorías comunes a partir de los descriptores, aunque se ha optado por omitir aquellas asignaturas marcadas con asterisco en la Tabla 2. En el caso de Cataluña, el documento no desarrolla descriptores, por lo que se ha recurrido a la ficha de cada asignatura ofrecida en la web de la Escuela Superior de Música de Cataluña (en adelante, ESMUC). En el currículum del País Vasco, los contenidos aparecen listados por especialidad y no por asignaturas, de modo que se han seleccionado los que se refieren a las tecnologías. Finalmente, en el caso de Baleares no se mencionan contenidos concretos.

Tabla 3. Contenidos de las asignaturas de Tecnología Musical según los planes de estudio

\begin{tabular}{|c|c|c|c|c|c|c|c|}
\hline & $\begin{array}{c}\text { Informática } \\
\text { del sonido }\end{array}$ & $\begin{array}{l}\text { Edición de } \\
\text { partituras }\end{array}$ & $\begin{array}{l}\text { Hardware/ } \\
\text { periféricos }\end{array}$ & $\begin{array}{c}\text { TIC } \\
\text { aplicadas a la } \\
\text { música }\end{array}$ & $\begin{array}{c}\text { Búsqueda y } \\
\text { difusión en red }\end{array}$ & $\begin{array}{c}\text { TIC } \\
\text { aplicadas } \\
\text { a la } \\
\text { educación }\end{array}$ & Multimedia \\
\hline Andalucía & $\mathrm{x}$ & $\mathrm{x}$ & $\mathrm{x}$ & $\mathrm{x}$ & & $\mathrm{x}$ & \\
\hline Asturias & $\mathrm{x}$ & $\mathrm{x}$ & & & $\mathrm{x}$ & & \\
\hline Canarias & $\mathrm{x}$ & $\mathrm{x}$ & & & $\mathrm{x}$ & $\mathrm{x}$ & $\mathrm{x}$ \\
\hline Cataluña & $\mathrm{x}$ & $\mathrm{x}$ & $\mathrm{x}$ & $\mathrm{x}$ & $\mathrm{x}$ & $\mathrm{x}$ & $\mathrm{x}$ \\
\hline $\begin{array}{c}\text { C. } \\
\text { Valenciana }\end{array}$ & $\mathrm{x}$ & $\mathrm{x}$ & & & $\mathrm{x}$ & & \\
\hline Galicia & $\mathrm{x}$ & $\mathrm{x}$ & & $\mathrm{x}$ & $\mathrm{x}$ & & \\
\hline Madrid & $\mathrm{x}$ & $\mathrm{x}$ & & & & $\mathrm{x}$ & \\
\hline Murcia & $\mathrm{x}$ & $\mathrm{x}$ & $\mathrm{x}$ & $\mathrm{x}$ & $\mathrm{x}$ & $\mathrm{x}$ & \\
\hline
\end{tabular}

@NURIA DÍEZ LATORRE. THE CONTENT OF THIS ARTICLE IS THE SOLE RESPONSIBILITY OF THE AUTHORS. THE REVISTA ELECTRÓNICA DE LEEME AND UNIVERSITAT DE VALĖNCIA ARE NOT LIABLE FOR ANY LEGAL ACTIONS THAT MAY ARISE INVOLVING THE ARTICLE'S CONTENT. REVISTA ELECTRÓNICA DE LEEME -LISTA ELECTRÓNICA EUROPEA DE MÚSICA EN LA EDUCACIÓN-HTTP://OJS.UV.ES/INDEX.PHP/LEEME/INDEX ISSN: 1575-9563 EDITORES: UNIVERSIDAD DE VALENCIA Y JESÚS TEJADA GIMÉNEZ. VISIBILIDAD DE ESTA REVISTA: SCOPUS, EMERGING SOURCES CITATION INDEX (CLARIVATE), EBSCO, CINDOC (CSIC), CITEFACTOR, COPAC, DIALNET, DICE (CSIC), DOAJ, E-REVISTAS (CSIC), EBSCO PREMIER, ERIH+, INSTITUCIONAL DE REDIRIS-CONSEJO SUPERIOR DE INVESTIGACIONES CIENTIFICAS Y ES DE ACCESO LIBRE. CREATIVE COMMONS LICENSE 4.0 


\begin{tabular}{cllllll}
\hline Navarra & $\mathrm{x}$ & $\mathrm{x}$ & & $\mathrm{x}$ & \\
\hline País Vasco & $\mathrm{x}$ & $\mathrm{x}$ & & $\mathrm{x}$ & $\mathrm{x}$ & \\
\hline Baleares & - & - & - & - & - & -
\end{tabular}

Fuente: Elaboración propia

Los contenidos más comunes son la formación técnica en software musical y también en redes para la búsqueda y difusión de contenidos (Figura 8). Los contenidos relativos a la educación corresponden, casi todos, a las asignaturas específicas de educación con TIC.

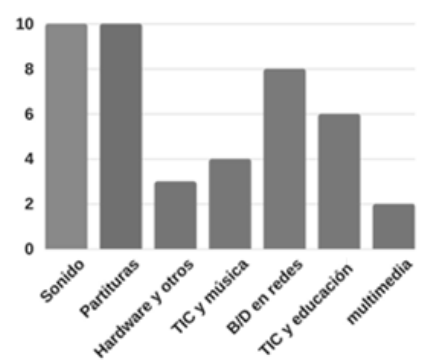

Figura 8. Frecuencia de los contenidos en los planes de estudio

Tabla 4. Contenidos relacionados con las TIC en materias de educación

\begin{tabular}{|c|c|c|}
\hline CONTENIDO & ASIGNATURA & PLAN \\
\hline \multirow{8}{*}{$\begin{array}{l}\text { La tecnología musical como recurso } \\
\text { didáctico }\end{array}$} & Didáctica de la música & Asturias \\
\hline & Didáctica del instrumento principal & \\
\hline & Didáctica del lenguaje musical & \\
\hline & Didáctica de la música en educación secundaria & Murcia \\
\hline & Didáctica de la música preescolar & \\
\hline & Didáctica del lenguaje musical & \\
\hline & Didáctica de los instrumentos musicales & \\
\hline & Didáctica de la educación musical & Canarias \\
\hline $\begin{array}{l}\text { La tecnología musical en la educación } \\
\text { secundaria }\end{array}$ & Didáctica de la música en la educación secundaria & \\
\hline \multirow{2}{*}{$\begin{array}{l}\text { Mecanismos para el registro: grabaciones } \\
\text { audiovisuales }\end{array}$} & Didáctica del instrumento/canto & \\
\hline & Didáctica del conjunto vocal e instrumental & \\
\hline $\begin{array}{l}\text { Nuevas tecnologías y medios de } \\
\text { comunicación }\end{array}$ & Planificación de aula y dinámicas educativas & Galicia \\
\hline \multirow{2}{*}{$\begin{array}{l}\text { Aplicación de nuevas tecnologías a la } \\
\text { educación musical }\end{array}$} & Organización, supervisión e innovación educativa & C. Valenciana \\
\hline & Organización y planificación de la enseñanza musical & Navarra \\
\hline
\end{tabular}

Fuente: Elaboración propia

Por otro lado, en los descriptores de las materias "Didáctica de la Educación Musical", "Fundamentos de Pedagogía" y "Organización Educativa" se han encontrado referencias muy escasas en relación con las TIC, por lo que no se ha estimado oportuno elaborar categorías. En su lugar, en la Tabla 4 se ha asociado cada contenido a las asignaturas en que aparece escrito de forma literal y a sus planes de estudio respectivos. Como puede observarse, las referencias a las tecnologías se localizan principalmente en asignaturas relacionadas con la Didáctica de la Educación Musical.

@NURIA DÍEZ LATORRE. THE CONTENT OF THIS ARTICLE IS THE SOLE RESPONSIBILITY OF THE AUTHORS. THE REVISTA ELECTRÓNICA DE LEEME AND UNIVERSITAT DE VALĖNCIA ARE NOT LIABLE FOR ANY LEGAL ACTIONS THAT MAY ARISE INVOLVING THE ARTICLE'S CONTENT. REVISTA ELECTRÓNICA DE LEEME -LISTA ELECTRÓNICA EUROPEA DE MÚSICA EN LA EDUCACIÓN-HTTP://OJS.UV.ES/INDEX.PHP/LEEME/INDEX ISSN: 1575-9563 EDITORES: UNIVERSIDAD DE VALENCIA Y JESÚS TEJADA GIMEENEZ. VISIBILIDAD DE ESTA REVISTA: SCOPUS, EMERGING SOURCES CITATION INDEX (CLARIVATE), EBSCO, CINDOC (CSIC), CITEFACTOR, COPAC, DIALNET, DICE (CSIC), DOAJ, E-REVISTAS (CSIC), EBSCO PREMIER, ERIH+, GALE CENGAGE LEARNING, IN-RECS, IRESIE, LATINDEX, MIAR, OCLC WORLDCAT, RESH, REDIB, RILM CORE JOURNALS, SUDOC, ULRICHS, ESTA REVISTA ESTÁ PUBLICADA CON EL APOYO INSTITUCIONAL DE REDIRIS-CONSEJO SUPERIOR DE INVESTIGACIONES CIENTIFICAS Y ES DE ACCESO LIBRE. CREATIVE COMMONS LICENSE 4.0 


\subsection{Análisis de las guías docentes}

En este análisis, se han examinado 102 documentos. En lo que respecta a las asignaturas de "Tecnología Musical", conviene señalar que faltan todas las guías de los conservatorios de Asturias, Baleares, Castellón y Musikene, así como la de "Tecnología Musical I" de Navarra. Se han agrupado los contenidos según 8 categorías: las 7 recogidas en la Tabla 3 más la "Acústica", que no está incluida dentro de la investigación, pero se ha estimado conveniente señalarla dado su peso en algunas guías.

1. Informática del sonido

3. Hardware y periféricos

5. Búsqueda y difusión en redes

7. Multimedia
2. Edición de partituras

4. TIC aplicadas a la música (análisis, estudio...)

6. TIC aplicadas a la educación

(8. Acústica)

Como puede observarse en la Tabla 5, los contenidos más frecuentes son las tecnologías relacionadas con el sonido (1) y la edición de partituras (2) que, además, son los más desarrollados en las guías docentes. Otro contenido mencionado con frecuencia es el de la búsqueda y difusión en redes (5), es decir, el uso de Internet, aunque apenas se desarrolla.

Tabla 5. Áreas de contenidos por asignaturas según las guías docentes (materia Tecnología Musical) Fuente: Elaboración propia

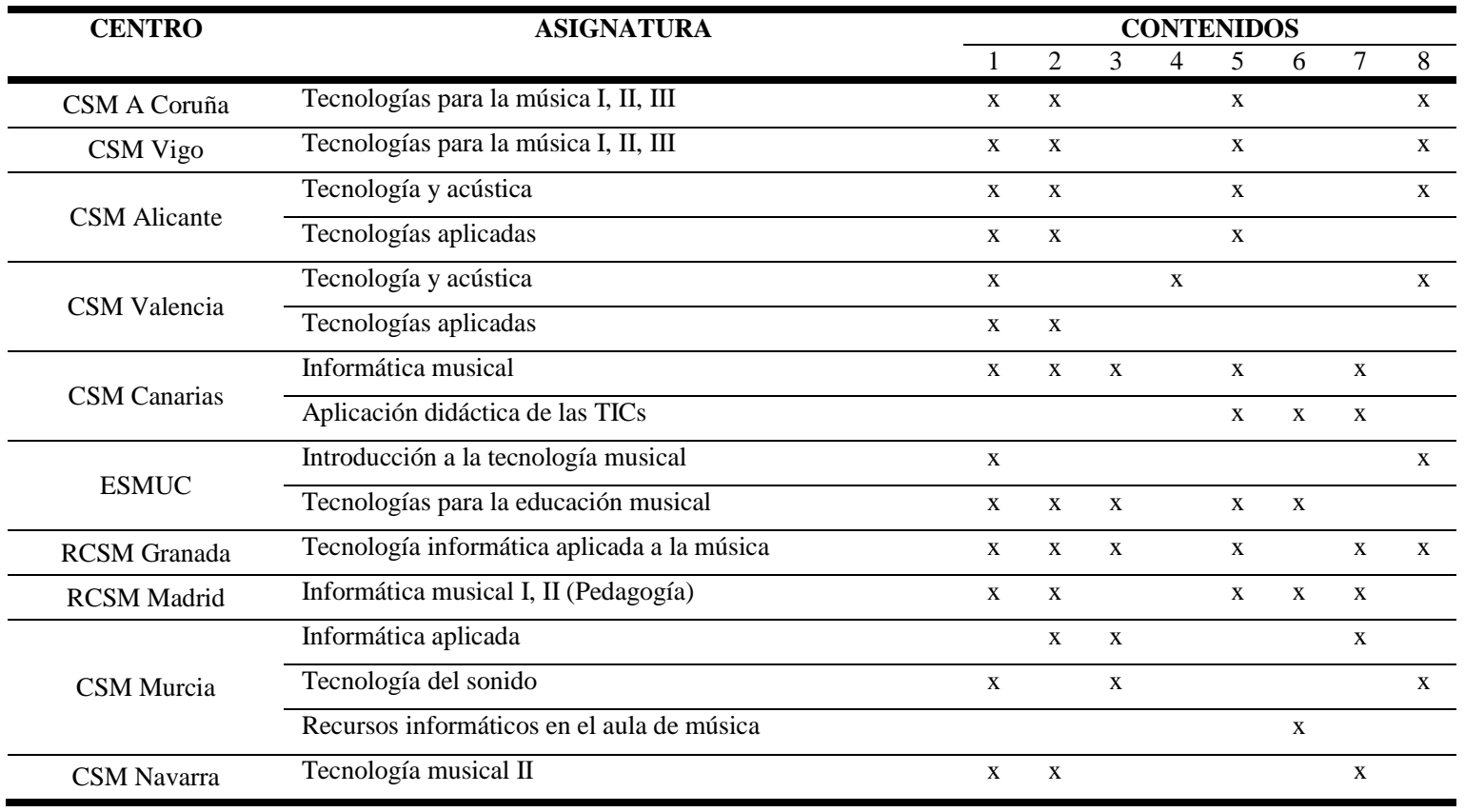

Fuente: Elaboración propia

Los contenidos relacionados con la educación (6) se encuentran sólo en las asignaturas específicas que hacen mención a ello en el título -Canarias, ESMUC y Murcia- y, en el caso de Madrid, aparecen como eje conductor en una asignatura que mezcla varios tipos de contenidos. En estos casos, la formación abarca herramientas más o menos diversas según el centro y algunos contenidos teóricos o relacionados con la reflexión sobre la práctica educativa: @NURIA DIEZZ LATORRE. THE CONTENT OF THIS ARTICLE IS THE SOLE RESPONSIBILITY OF THE AUTHORS. THE REVISTA ELECTRÓNICA DE LEEME AND UNIVERSITAT DE VALĖNCIA ARE
NOT LIABLE FOR ANY LEGAL ACTIONS THAT MAY ARISE INVOLVING THE ARTICLE'S CONTENT. REVISTA ELECTRÓNICA DE LEEME -LISTA ELECTRÓNICA EUROPEA DE MÚSICA EN LA EDUCACIÓN-HTTP://OJSUVVES/INDEXPHP/LEEME/INDEXISSN: 1575-9563 EDITORES: UNIVERSIDAD DE VALENCIA Y JESÚS TEJADA GIMÉNEZ. VISIBILIDAD DE ESTA REVISTA: SCOPUS, EMERGING SOURCES CITATION INDEX (CLARIVATE), EBSCO, CINDOC (CSIC), CITEFACTOR, COPAC, DIALNET, DICE (CSIC), DOAJ, E-REVISTAS (CSIC), EBSCO PREMIER, ERIH+, GALE CENGAGE LEARNING, IN-RECS, IRESIE, LATINDEX, MIAR, OCLC WORLDCAT, RESH, REDIB, RILM CORE JOURNALS, SUDOC, ULRICHS, ESTA REVISTA ESTÁ PUBLICADA CON EL APOYO INSTITUCIONAL DE REDIRIS-CONSEJO SUPERIOR DE INVESTIGACIONES CIENTIFICAS Y ES DE ACCESO LIBRE. CREATIVE COMMONS LICENSE 4.0 
- Empleo de editores de sonido, partituras y multimedia en general para elaborar proyectos y materiales didácticos musicales.

- Herramientas de entrenamiento de destrezas y apoyo a la creatividad.

- Uso de la pizarra digital y de otros dispositivos.

- Recursos de la Web 2.0, repositorios de contenidos, redes sociales, aplicaciones diversas, desarrollo de e-portafolios y entornos personales de aprendizaje.

- Contenidos teóricos y reflexivos acerca de la innovación educativa con las TIC y su importancia en la formación del profesorado. Modelos teóricos.

Por otro lado, en las asignaturas vinculadas a materias educativas se menciona a las TIC en las competencias, pero mínimamente en el resto de elementos. La Tabla 6 muestra las referencias de contenidos que vienen asociados, por lo general, a las asignaturas de didáctica, aunque también a ciertas asignaturas de las otras dos materias. En ocasiones, apenas se concreta más allá del título del bloque. En cuanto a la metodología, sólo 3 centros especifican el uso de las TIC en el desarrollo de las clases: algunos de los recursos citados son la pizarra digital, sistemas de gestión del aprendizaje y herramientas de comunicación y trabajo colaborativo. Con respecto a la evaluación el uso de tecnologías se valora, generalmente, en la elaboración de trabajos y como apoyo a las exposiciones orales.

Tabla 6. Contenidos relacionados con las TIC en las guías docentes de materias educativas (fuente: elaboración propia).

\begin{tabular}{|c|c|c|}
\hline CENTRO & ASIGNATURA & ELEMENTO \\
\hline RCSM Granada & Técnicas de investigación musical & $\begin{array}{l}\text { Archivos. Bibliotecas. Centros de documentación. } \\
\text { Internet. Cómo localizar la información. }\end{array}$ \\
\hline CSM Canarias & Didáctica específica & $\begin{array}{l}\text { La tecnología musical en la Educación Secundaria y el } \\
\text { Bachillerato }\end{array}$ \\
\hline \multirow[t]{2}{*}{ ESMUC } & Didáctica general & $\begin{array}{l}\text { Los recursos didácticos y las nuevas tecnologías aplicadas } \\
\text { a la enseñanza y aprendizaje de la música. }\end{array}$ \\
\hline & Pedagogía sistemática de la música II & Los medios de comunicación y las nuevas tecnologías. \\
\hline CSM A Coruña & Técnicas de aula II & $\begin{array}{l}\text { La aplicación de las TIC en la enseñanza de música. Los } \\
\text { cambios en el escenario educativo. }\end{array}$ \\
\hline CSM Vigo & Planificación de aula y dinámicas educativas & $\begin{array}{l}\text { La creación de entornos de aprendizaje: organización, } \\
\text { estrategias. Nuevas tecnologías. }\end{array}$ \\
\hline RCSM Madrid & Fundamentos de Pedagogía I y II & $\begin{array}{l}\text { Nuevos ámbitos de la educación. Virtualización de la } \\
\text { docencia: b-learning, e-learning, Miríada X, redes sociales } \\
\text { y comunidades virtuales, mini-vídeos docentes (MOOC), } \\
\text { plataforma Moodle, etc. }\end{array}$ \\
\hline CSM Murcia & $\begin{array}{l}\text { Didáctica de la música en educación } \\
\text { secundaria I }\end{array}$ & $\begin{array}{l}\text { Estrategias para integrar las TIC en los procesos de } \\
\text { enseñanza y aprendizaje musical. }\end{array}$ \\
\hline \multirow[t]{2}{*}{ CSM Castellón } & Didáctica general de la música II & Tecnología educativa y formación del profesorado. \\
\hline & $\begin{array}{l}\text { Organización, supervisión e innovación } \\
\text { educativa II }\end{array}$ & $\begin{array}{l}\text { Innovación tecnológica en educación musical: aplicación } \\
\text { de nuevas tecnologías en la educación musical, recursos y } \\
\text { materiales para el aula de música (partituras gráficas), } \\
\text { Soundcool como herramienta de composición musical } \\
\text { colaborativa. }\end{array}$ \\
\hline CSM Valencia & Didáctica general de la música II & $\begin{array}{l}\text { Tecnología educativa, modelo TPACK y formación del } \\
\text { profesorado }\end{array}$ \\
\hline
\end{tabular}

Fuente: Elaboración propia

\section{Conclusiones}

El Real Decreto 631/2010 establece un mínimo de 6 créditos ECTS destinados a la "Tecnología Musical" para la especialidad de Pedagogía Musical, cantidad que se supera en la

@NURIA DÍEZ LATORRE. THE CONTENT OF THIS ARTICLE IS THE SOLE RESPONSIBILITY OF THE AUTHORS. THE REVISTA ELECTRÓNICA DE LEEME AND UNIVERSITAT DE VALĖNCIA ARE NOT LIABLE FOR ANY LEGAL ACTIONS THAT MAY ARISE INVOLVING THE ARTICLE'S CONTENT. REVISTA ELECTRÓNICA DE LEEME -LISTA ELECTRÓNICA EUROPEA DE MÚSICA EN LA EDUCACIÓN-HTTP://OJS.UV.ES/INDEX.PHP/LEEME/INDEX ISSN: 1575-9563 EDITORES: UNIVERSIDAD DE VALENCIA Y JESÚS TEJADA GIMÉNEZ. VISIBILIDAD DE ESTA REVISTA: SCOPUS, EMERGING SOURCES CITATION INDEX (CLARIVATE), EBSCO, CINDOC (CSIC), CITEFACTOR, COPAC, DIALNET, DICE (CSIC), DOAJ, E-REVISTAS (CSIC), EBSCO PREMIER, ERIH+, INSTITUCIONAL DE REDIRIS-CONSEJO SUPERIOR DE INVESTIGACIONES CIENTIFICAS Y ES DE ACCESO LIBRE. CREATIVE COMMONS LICENSE 4.0 
mayoría de planes de estudio. Sin embargo, algunos de ellos incluyen dentro de esta materia ciertas asignaturas que no implican necesariamente la presencia de las TIC, hecho que se ha podido comprobar tras el examen de sus contenidos. Sin considerar dichas asignaturas, el número de créditos relacionados directamente con las TIC se reduce en casi todos los planes de estudio: desde 3 hasta 11 créditos, según la comunidad autónoma, de los 240 que componen la titulación. El número de asignaturas oscila entre 1 y 3 , y sólo 5 planes dedican una a las tecnologías aplicadas a la educación. En el resto de casos, las asignaturas de esta materia no incluyen contenidos relacionados con la didáctica y las TIC, sino que se centran en el conocimiento y uso de herramientas relacionadas con el sonido y con la edición de partituras: se trata de una formación común al resto de especialidades y no da respuesta a las necesidades añadidas que conlleva la función docente. La carencia de asignaturas de perfil tecnológico-educativo se suple en algunos planes mediante la integración de contenidos vagamente definidos en otras asignaturas de la especialidad; en otros, no hay ninguna referencia a la educación con las TIC.

De esta investigación se desprende que la formación en TIC adecuada a la futura profesión docente todavía tiene escasa consideración en los conservatorios superiores. Su presencia en los distintos currículos es desigual: algunos planes cuentan con asignaturas específicas, otros añaden contenidos transversales y unos cuantos, directamente, no contemplan el aspecto educativo de las TIC. De cara a futuras reformas de planes de estudios, se debería considerar una formación más equitativa en este sentido. Quizás una importante revisión debería ser una mayor concreción en los descriptores de las materias para Pedagogía Musical, ya que el único elemento relacionado con las tecnologías aparece en la materia "Didáctica de la Educación Musical" y cita únicamente "la tecnología musical como recurso didáctico". Este epígrafe se refiere a la tecnología específicamente musical y no implica, necesariamente, los recursos, herramientas y modelos teóricos necesarios para promover una verdadera innovación educativa. En este sentido, coincidimos con Losada, Valverde y Correa (2012) y Fernández, Fernández y Cebreiro (2016) en que se precisa una formación en TIC más pedagógica y no un mero adiestramiento en recursos tecnológicos.

En la formación del profesorado no se puede pasar por alto la cultura tecnológica en que está inmersa la sociedad. El futuro docente debe ser capaz de pensar formas alternativas a la enseñanza tradicional, idea que compartimos con Sancho, Bosco, Alonso y Sánchez (2015). Desde esta perspectiva, resulta necesario revalorizar el papel de las TIC en la elaboración de los planes de estudio de Pedagogía Musical de los conservatorios superiores. Las nuevas tecnologías forman parte de la realidad y no se las puede mantener al margen en los procesos de enseñanzaaprendizaje, incluidos los de la música. Si no se les otorga un espacio suficiente en los currículos formativos del profesorado, es probable que, en muchos casos, se las acabe considerando como un mero complemento, quizás prescindible, lejos de valorarlas como un motor de cambio, innovación y mejora de las posibilidades educativas.

\section{Referencias}

Ballesta, J. y Céspedes, R. (2015). Los contenidos de Tecnología Educativa en las titulaciones de grado de las universidades españolas. RELATEC: Revista Latinoamericana de Tecnología Educativa, 14 (1), 133-144. doi: 10.17398/1695-288X.14.1.133

@NURIA DÍEZ LATORRE. THE CONTENT OF THIS ARTICLE IS THE SOLE RESPONSIBILITY OF THE AUTHORS. THE REVISTA ELECTRÓNICA DE LEEME AND UNIVERSITAT DE VALĖNCIA ARE NOT LIABLE FOR ANY LEGAL ACTIONS THAT MAY ARISE INVOLVING THE ARTICLE'S CONTENT. REVISTA ELECTRÓNICA DE LEEME - LISTA ELECTRÓNICA EUROPEA DE MÚSICA EN LA EDUCACIÓN-HTTP://OJS.UV.ES/INDEX.PHP/LEEME/INDEX ISSN: 1575-9563 EDITORES: UNIVERSIDAD DE VALENCIA Y JESÚS TEJADA GIMÉNEZ. VISIBILIDAD DE ESTA REVISTA: SCOPUS, EMERGING SOURCES CITATION INDEX (CLARIVATE), EBSCO, CINDOC (CSIC), CITEFACTOR, COPAC, DIALNET, DICE (CSIC), DOAJ, E-REVISTAS (CSIC), EBSCO PREMIER, ERIH+, GALE CENGAGE LEARNING, IN-RECS, IRESIE, LATINDEX, MIAR, OCLC WORLDCAT, RESH, REDIB, RILM CORE JOURNALS, SUDOC, ULRICHS, ESTA REVISTA ESTÁ PUBLICADA CON EL APOYO INSTITUCIONAL DE REDIRIS-CONSEJO SUPERIOR DE INVESTIGACIONES CIENTIFICAS Y ES DE ACCESO LIBRE. CREATIVE COMMONS LICENSE 4.0 
Balo, M., Lago, P. y Ponce de León, L. (2014). Los alumnos ante el dictado musical. Las TIC como aliadas para mejorar las experiencias. Didáctica, Innovación y Multimedia, 28, 1-14. Recuperado de https://ddd.uab.cat/

Bardin, L. (1996). Análisis de contenido. Madrid: Akal.

Belló, J. y González, M. (2010, junio). Las utilidades didácticas de internet y e-learning en los conservatorios de música de Galicia: Usos y actitudes del profesorado. Comunicación presentada en Seminario Internacional de Investigación en Educación Musical, Universidad Complutense de Madrid, Madrid.

Bernabé, M. y Azorín, J. (2015). La pizarra digital en la clase de lenguaje musical: aprendizaje armónico-analítico. Opción, 31, 235-249. Recuperado de http://www.redalyc.org/

Berrón, E., Balsera, F.J. y Monreal, I. M. (2017). Ansiedad de los alumnos en la asignatura de Lenguaje Musical y estrategias para reducirla. Revista Internacional de Educación Musical, 5, 41-48. doi: 10.12967/RIEM-2017-5-p041-048

Colás, P. y Hernández, G. (2014). Incidencia de la formación del profesorado en sus creencias sobre el valor de las TIC en la enseñanza de la música. Educatio Siglo XXI, 32 (3), 51-74. doi: $10.6018 / \mathrm{j} / 210981$

Coll, C. (2009). Aprender y enseñar con las TIC. Expectativas, realidad y potencialidades. En R. Carneiro, J. C. Toscano y T. Díaz (Coords.). Los desafíos de las TIC para el cambio educativo (pp. 113-126). Madrid: Santillana.

De la Rosa, E. (2015). El uso de las TIC en los centros superiores de música. Un análisis desde la perspectiva del profesorado, del alumnado y de los recursos de las webs institucionales (Tesis doctoral). Universidad de las Palmas de Gran Canaria, Las Palmas de Gran Canaria. Recuperado de http://cris.ulpgc.es/

Fernández, J. C., Fernández, M. C. y Cebreiro, B. (2016). Competencias en TIC del profesorado en Galicia: variables que inciden en las necesidades formativas. Innovación educativa, 26, $215-$ 231. doi: $10.15304 /$ ie. 26.3256

Galera, M., Tejada, J. y Trigo, E. (2013). El editor de partituras como medio para facilitar el estudio de la lectura musical cantada. Electronic Journal of Research in Educational Psychology, 29, 215-238. Recuperado de https://idus.us.es

Gutiérrez, A., Palacios, A. y Torrego, L. (2010). La formación de los futuros maestros y la integración de las TIC en la educación: anatomía de un desencuentro. Revista de educación, 352, 267-293. Recuperado de http://www.revistaeducacion.educacion.es/

Losada, D., Valverde, J. y Correa, J. M. (2012). La tecnología educativa en la universidad pública española. Pixel-Bit: Revista de medios y educación, 41, 133-148. doi: 10.17398/1695288X.14.1.101 @NURIA DIEZZ LATORRE. THE CONTENT OF THIS ARTICLE IS THE SOLE RESPONSIBIIITY OF THE AUTHORS. THE REVISTA ELECTRÓNICA DE LEEME AND UNIVERSITAT DE VALĖNCIA ARE
NOT LIABLE FOR ANY LEGAL ACTIONS THAT MAY ARISE INVOLVING THE ARTICLE'S CONTENT. REVISTA ELECTRÓNICA DE LEEME -LISTA ELECTRÓNICA EUROPEA DE MÚSICA EN LA EDUCACIÓN-HTTP://OJS.UV.ES/INDEX.PHP/LEEME/INDEX ISSN: 1575-9563 EDITORES: UNIVERSIDAD DE VALENCIA Y JESÚS TEJADA GIMÉNEZ. VISIBILIDAD DE ESTA REVISTA: SCOPUS, EMERGING SOURCES CITATION INDEX (CLARIVATE), EBSCO, CINDOC (CSIC), CITEFACTOR, COPAC, DIALNET, DICE (CSIC), DOAJ, E-REVISTAS (CSIC), EBSCO PREMIER, ERIH+, GALE CENGAGE LEARNING, IN-RECS, IRESIE, LATINDEX, MIAR, OCLC WORLDCAT, RESH, REDIB, RILM CORE JOURNALS, SUDOC, ULRICHS, ESTA REVISTA ESTÁ PUBLICADA CON EL APOYO INSTITUCIONAL DE REDIRIS-CONSEJO SUPERIOR DE INVESTIGACIONES CIENTIFICAS Y ES DE ACCESO LIBRE. CREATIVE COMMONS LICENSE 4.0 
Martínez, I. (2017). La educación musical donde no llegan las palabras. Implementación de un blog colaborativo como herramienta en las asignaturas grupales de conservatorio. Experiencias educativas, 16, 70-91. Recuperado de http://www.e-revistes.uji.es

Ministerio de Educación (2010). Real Decreto 631/2010, de 14 de mayo, por el que se regula el contenido básico de las Enseñanzas Artísticas Superiores de Grado en Música establecidas en la Ley Orgánica 2/2006, de 3 de mayo, de Educación. BOE (05/06/2010), nº 137, pp. 48480-48500.

Moreno, A. J. (2018). Las TIC en el proceso de enseñanza y aprendizaje. En M. L. Cacheiro (Ed.). Educación y tecnología: estrategias didácticas para la integración de las TIC (pp. 9-39). Madrid: UNED.

Ordoñana, J. A., Laucirica, A. y Tejada, J. (2004). Estudio cualitativo sobre el uso de programas informáticos para el desarrollo de destrezas rítmicas en la enseñanza musical especializada. Revista de Psicodidáctica, 17, 127-136. Recuperado de http://www.ehu.eus/

Paredes, J., Guitert, M. y Rubia, B. (2015). La innovación y la tecnología educativa como base de la formación inicial del profesorado para la renovación de la enseñanza. RELATEC: Revista Latinoamericana de Tecnología Educativa 14 (1), 101-114. doi: 10.17398/1695-288X.14.1.101

Sancho, J. M., Bosco, A., Alonso, C. y Sánchez, J. A. (2015). Formación del profesorado en Tecnología Educativa: de cómo las realidades generan los mitos. RELATEC: Revista Latinoamericana de Tecnología Educativa, 14 (1), 17-30. doi: 10.17398/1695-288X.14.1.17

Silva, J. y Astudillo, A. (2012). Inserción de TIC en la formación inicial docente: barreras y oportunidades. Revista Iberoamericana de Educación, 58 (4), 1-12. Recuperado de https://rieoei.org/

Tejada, J. (2010). Diseño de un modelo de adiestramiento rítmico mediante programas informáticos en conservatorios de música. En J. M. Pérez Tornero, J. Cabero Almenara y L. Vilches (Coords.). Actas del Congreso Euro-Iberoamericano "Alfabetización Mediática y Culturas Digitales" (pp. 866-885). Sevilla: Universidad de Sevilla, Gabinete Comunicación y Educación. Recuperado de https://idus.us.es/

Timiraos, E. (2015). Uso y valoración de las TIC en el aprendizaje musical por los alumnos de los conservatorios de música profesional y superior de A Coruña: estudio de caso de uso de "IrealB" y "Band in a Box" en el aula de improvisación (Tesis doctoral inédita). Universidad Nacional de Educación a Distancia, Madrid. Recuperado de: http://e-spacio.uned.es/

@NURIA DÍEZ LATORRE. THE CONTENT OF THIS ARTICLE IS THE SOLE RESPONSIBILITY OF THE AUTHORS. THE REVISTA ELECTRÓNICA DE LEEME AND UNIVERSITAT DE VALĖNCIA ARE NOT LIABLE FOR ANY LEGAL ACTIONS THAT MAY ARISE INVOLVING THE ARTICLE'S CONTENT. REVISTA ELECTRÓNICA DE LEEME -LISTA ELECTRÓNICA EUROPEA DE MÚSICA EN LA EDUCACIÓN-HTTP://OJS.UV.ES/INDEX.PHP/LEEME/INDEX ISSN: 1575-9563 EDITORES: UNIVERSIDAD DE VALENCIA Y JESÚS TEJADA GIMÉNEZ. VISIBILIDAD DE ESTA REVISTA: SCOPUS, EMERGING SOURCES CITATION INDEX (CLARIVATE), EBSCO, CINDOC (CSIC), CITEFACTOR, COPAC, DIALNET, DICE (CSIC), DOAJ, E-REVISTAS (CSIC), EBSCO PREMIER, ERIH+, INSTITUCIONAL DE REDIRIS-CONSEJO SUPERIOR DE INVESTIGACIONES CIENTIFICAS Y ES DE ACCESO LIBRE. CREATIVE COMMONS LICENSE 4.0 


\section{Anexos}

Tabla con análisis de las guías docentes (curso 2017-2018). Asignaturas de las materias educativas:

- Didáctica de la Educación Musical (DEM).

- Fundamentos de Pedagogía (FP).

- Organización Educativa (OE).

\begin{tabular}{|c|c|c|c|c|c|c|c|c|c|c|c|c|c|}
\hline \multirow{2}{*}{ CENTRO } & \multirow{2}{*}{ ASIGNATURA } & \multirow{2}{*}{ Materia } & \multicolumn{3}{|c|}{ Competencias } & \multirow[t]{2}{*}{ Resultados } & \multirow[t]{2}{*}{ Objetivos } & \multirow[t]{2}{*}{ Contenidos } & \multirow[t]{2}{*}{ Metodología } & \multicolumn{4}{|c|}{ Evaluación } \\
\hline & & & $\mathbf{T}$ & G & EP & & & & & CE & PE & IE & CC \\
\hline \multirow{8}{*}{$\begin{array}{c}\text { RCSM } \\
\text { Granada }\end{array}$} & Didáctica de la música I, II y III & DEM & $x$ & $x$ & $\mathrm{x}$ & & & & & $x$ & & & \\
\hline & Composición aplicada a la didáctica I y II & DEM & $\mathrm{x}$ & $\mathrm{x}$ & & & & & & $x$ & & & $\mathrm{x}$ \\
\hline & Pedagogía de la música I y II & FP & $x$ & & $x$ & & & & & $x$ & & & $x$ \\
\hline & Metodología de la investigación pedagógica & FP & & & & & & & & $x$ & & & $x$ \\
\hline & Técnicas de investigación musical & FP & $x$ & $\mathrm{x}$ & $\mathrm{x}$ & & & $\mathrm{x}$ & & $x$ & & & \\
\hline & Música y movimiento & FP & & & $x$ & & & & & & & & \\
\hline & Organización educativa & $\mathrm{OE}$ & $x$ & & & & & & & $x$ & & & $x$ \\
\hline & Psicología del desarrollo y de la educación & $\mathrm{OE}$ & $\mathrm{x}$ & & $x$ & & & & & $\mathrm{x}$ & & & \\
\hline \multirow{9}{*}{$\begin{array}{l}\text { CSM del } \\
\text { Principad } \\
\text { o de } \\
\text { Asturias }\end{array}$} & Didáctica de la música I y II & DEM & $(x)$ & $(x)$ & $(x)$ & & & $(x)$ & & & & & \\
\hline & Didáctica del instrumento principal & DEM & & $(x)$ & (x) & & & (x) & & & & & \\
\hline & Didáctica del lenguaje musical & DEM & (x) & $(x)$ & (x) & & & $(\mathrm{x})$ & & & & & \\
\hline & Metodología de la investigación & FP & $(x)$ & & & & & & & & & & \\
\hline & Psicología del desarrollo y de la educación & FP & (x) & $(x)$ & & & & & & & & & \\
\hline & Sociología e historia de la educación & FP & (x) & & & & & & & & & & \\
\hline & Diagnóstico y evaluación en la educación & $\mathrm{OE}$ & $(x)$ & $(x)$ & $(x)$ & & & & & & & & \\
\hline & Organización y gestión de centros & $\mathrm{OE}$ & $(x)$ & $(x)$ & & & & & & & & & \\
\hline & Orientación educativa & $\mathrm{OE}$ & $(\mathrm{x})$ & $(x)$ & & & & & & & & & \\
\hline
\end{tabular}

@NURIA DiEZ LATORRE. THE CONTENT OF THIS ARTICLE IS THE SOLE RESPONSIBLLITY OF THE AUTHORS. THE REVISTA ELECTRÓNICA DE LEEME AND UNIVRSITAT DE VALĖNCIA ARE NOT LIABLE FOR ANY LEGAL ACTIONS THAT MAY ARISE INVOLVING THE ARTICLE'S CONTENT. REVISTA

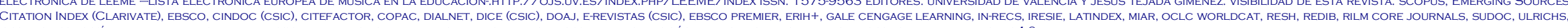
ESTA REVISTA ESTA PUBLICADA CON EL APOYO INSTITUCIONAL DE REDIRISCOONSEJO SUPERIOR DE INVESTIGACIONES CIENTIFICAS Y ES DE ACCESO LIBRE. CREATIVE COMMONS LICENSE 4.O 


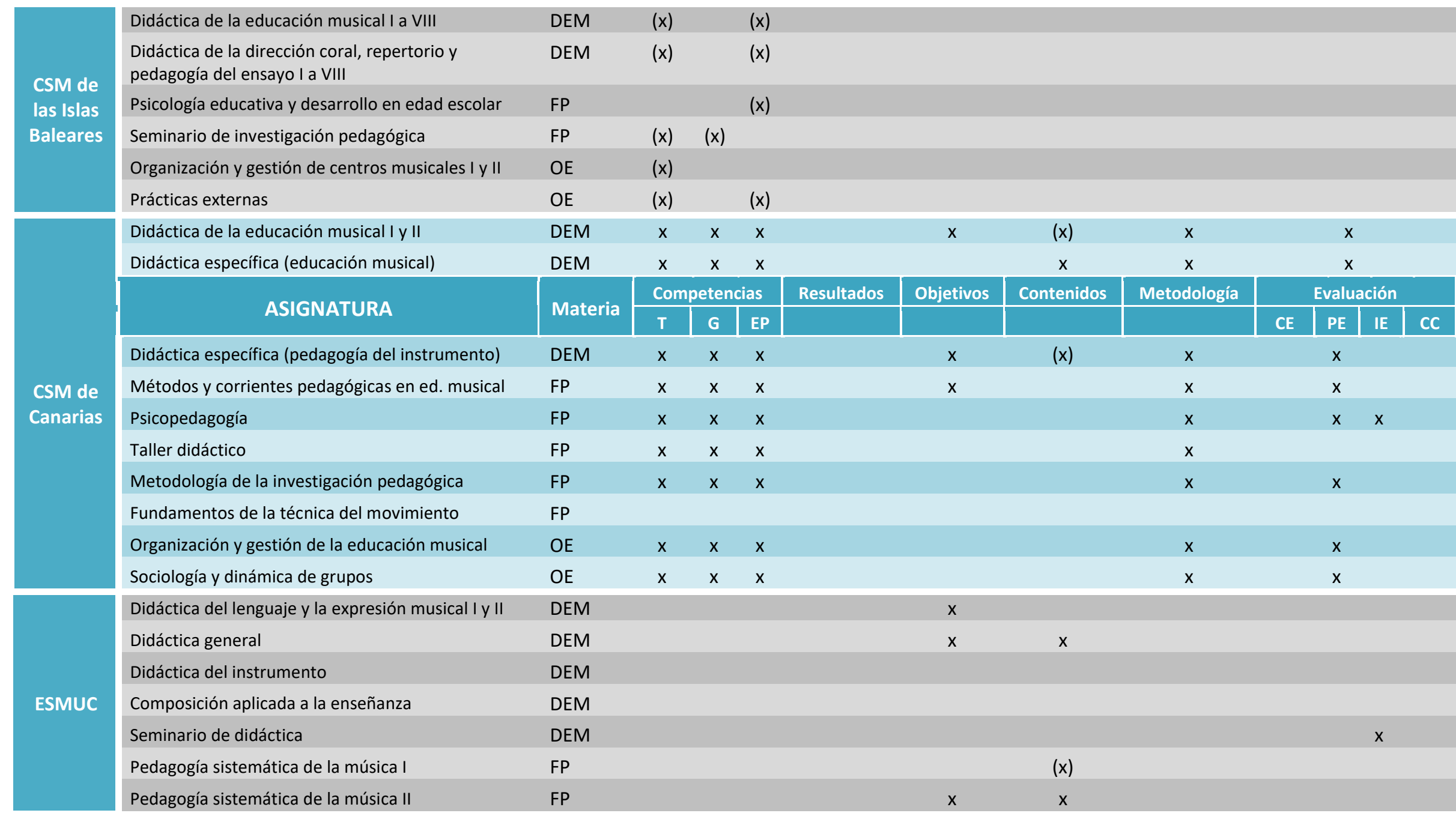

@NURIA DiEZ LATORRE. THE CONTENT OF THIS ARTICLE IS THE SOLE RESPONSIBLLITY OF THE AUTHORS. THE REVISTA ELECTRÓNICA DE LEEME AND UNIVRSITAT DE VALĖNCIA ARE NOT LIABLE FOR ANY LEGAL ACTIONS THAT MAY ARIIE INVOLVING THE ARTICLE'S CONTENT. REVISTA CITATION INDEX (CLARIVATE), EBSCO, CINDOC (CSIC), CITEFACTOR, COPAC, DIALNET, DICE (CSIC), DOAJ, EREVIITAS (CSIC), EBSCO PREMIER, ERIH+, GALE CENGAGE LEARNING, IN-RECS, IRESIE, LATINDEX, MIAR, OCLCL WORLDCAT, RESH, REDIB, RLLM CORE JOURNALS, SUDOC, ULRICHS, ESTA REVISTA ESTA PUBLICADA CON EL APOYO INSTTIUCIONAL DE REDIRISCONSEJO SUPERIOR DE INVESTIGACIONES CIENTIFICAS Y ES DE ACCESO LIBRE. CREATIVE COMMONS LICENSE 4.O 


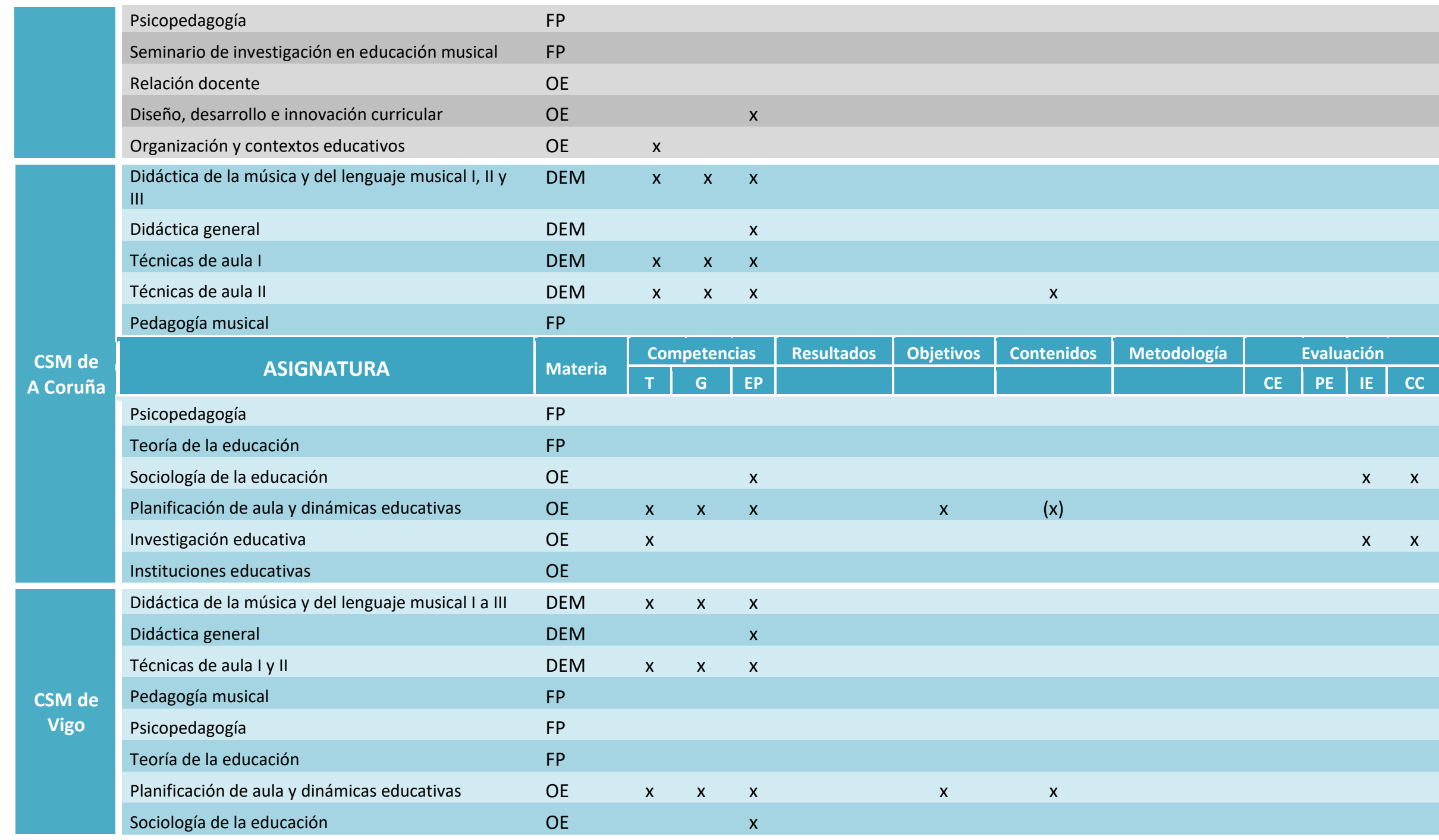

@NURIA DIEZ LATORRE. THE CONTENT OF THIS ARTICLE IS THE SOLE RESPONSIBLITY OF THE AUTHORS. THE REVISTA ELECTRÓNICA DE LEEME AND UNIVERSITAT DE VALENNCIA ARE NOT LIABLE FOR ANY LEGAL ACTIONS THAT MAY ARISE INVOLVING THE ARTICLE'S CONTENT. REVISTA CITATION INDEX (CLARIVATE), EBSCO, CINDOC (CSIC), CITEFACTOR, COPAC, DIALNET, DICE (CSIC), DOAJ, EREVIITAS (CSIC), EBSCO PREMIER, ERIH+, GALE CENGAGE LEARNING, IN-RECS, IRESIE, LATINDEX, MIAR, OCLC WORLDCAT, RESH, REDIB, RLLM CORE JOURNALS, SUDOC, ULRICHS, ESTA REVISTA ESTA PUBLICADA CON EL APOYO INSTITUCIONAL DE REDIRISCOONSEJO SUPERIOR DE INVESTIGACIONES CIENTIFICAS Y ES DE ACCESO LIBRE. CREATIVE COMMONS LICENSE 4.O 


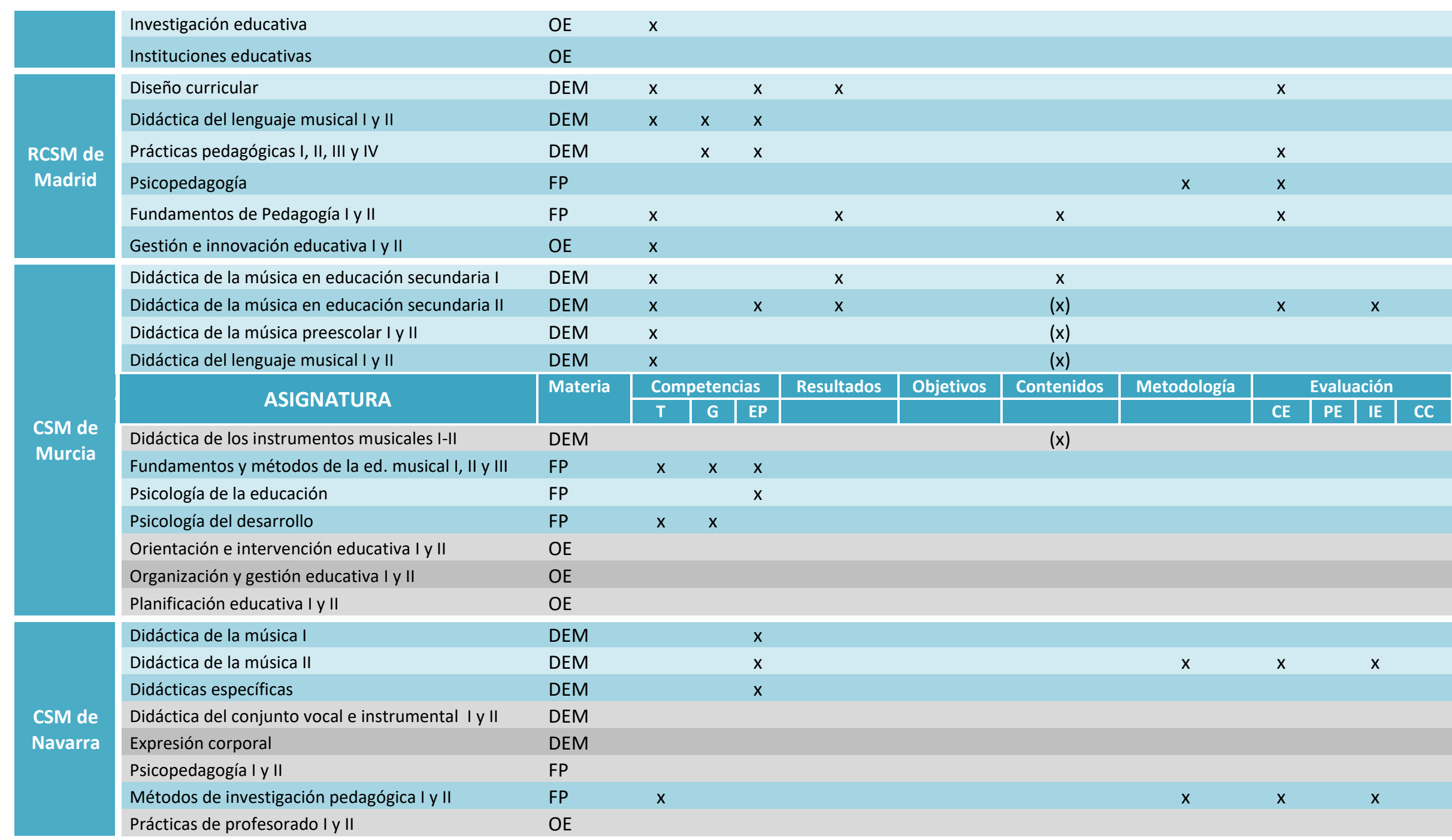

@NURIA DiEZ LATORRE. THE CONTENT OF THIS ARTICLE IS THE SOLE RESPONSIBLITY OF THE AUTHORS. THE REVISTA ELECTRÓNICA DE LEEME AND UNIVERSTTAT DE VALËNCIA ARE NOT LIABLE FOR ANY LEGAL ACTIONS THAT MAY ARISE INVOLVING THE ARTICLE'S CONTENT. REVISTA CITATION INDEX (CLARIVATE), EBSCO, CINDOC (CSIC), CITEFACTOR, COPAC, DIALNET, DICE (CSIC), DOAJ, EREVIITAS (CSIC), EBSCO PREMIER, ERIH+, GALE CENGAGE LEARNING, IN-RECS, IRESIE, LATINDEX, MIAR, OCLC WORLDCAT, RESH, REDIB, RLLM CORE JOURNALS, SUDOC, ULRIIHS, ESTA REVISTA ESTÁ PUBLICADA CON EL APOYO INSTTTUCIONAL DE REDIRISCONSEJO SUPERIOR DE INVESTIGACIONES CIENTIFICAS Y ES DE ACCESO LIBRE. CREATIVE COMMONS LICENSE 4.O 


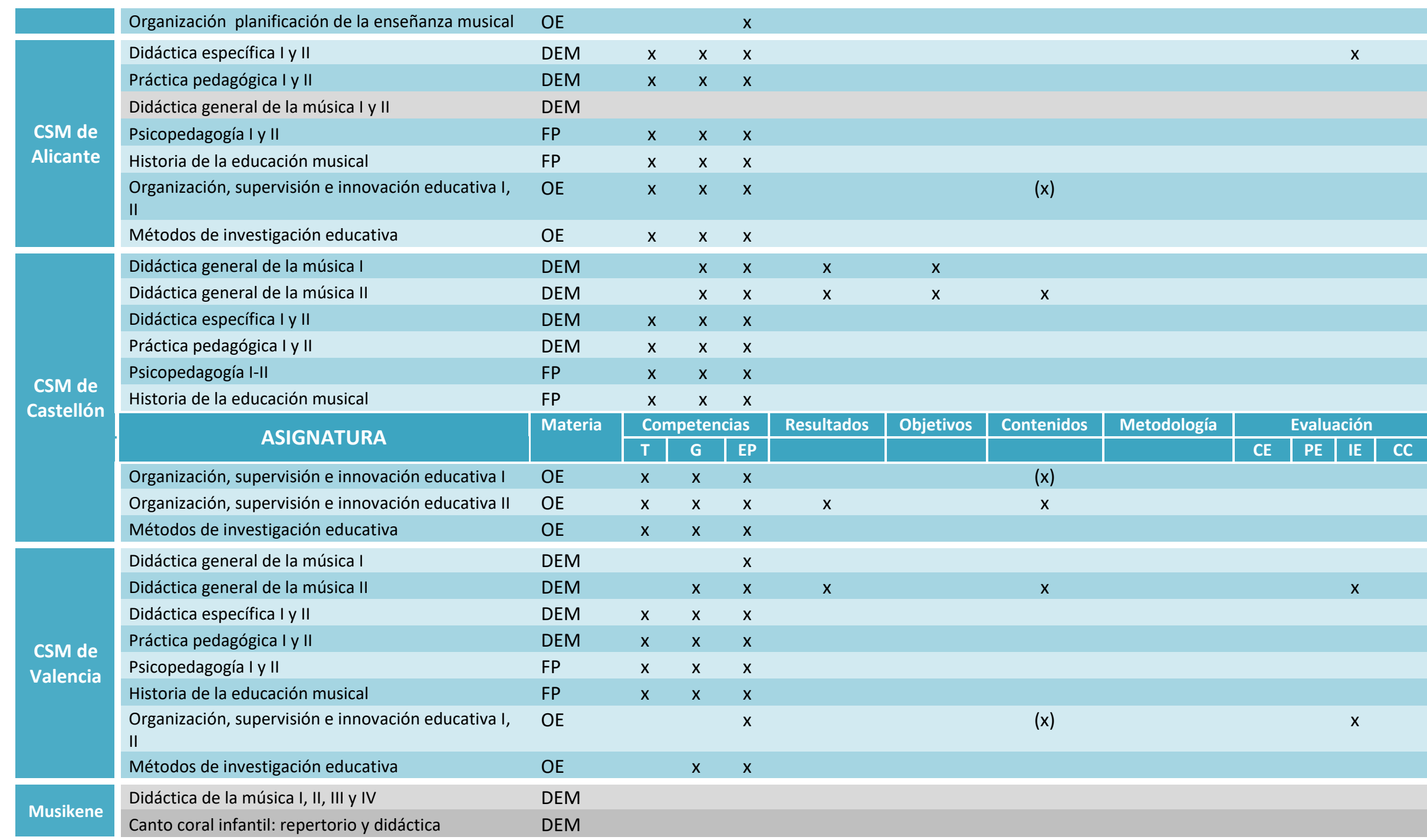

@NURIA DIEZ LATORRE. THE CONTENT OF THIS ARTICLE IS THE SOLE RESPONSIBLITY OF THE AUTHORS. THE REVISTA ELECTRÓNICA DE LEEME AND UNIVERSITAT DE VALĖNCIA ARE NOT LIABLE FOR ANY LEGAL ACTIONS THAT MAY ARISE INVOLVING THE ARTICLE'S CONTENT. REVISTA CITATION INDEX (CLARIVATE), EBSCO, CINDOC (CSIC), CITEFACTOR, COPAC, DIALNET, DICE (CSIC), DOAJ, EREVISTAS (CSIC), EBSCO PREMIER, ERIH+, GALE CENGAGE LEARNING, IN-RECS, IRESIE, LATINDEX, MIAR, OCLC WORLDCAT, RESH, REDIB, RLLM CORE JOURNALS, SUDOC, ULRICHS, ESTA REVISTA ESTÁ PUBLICADA CON EL APOYO INSTITUCIONAL DE REDIRISCONSEJO SUPERIOR DE INVESTIGACIONES CIENTIFICAS Y ES DE ACCESO LIBRE. CREATIVE COMMONS LICENSE 4.O 


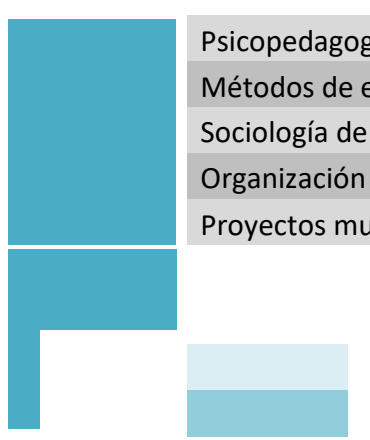

Con acceso a GD
Sin acceso a GD*
FP
FP
OE
OE
OE

$\mathrm{x}=$ elemento encontrado en la GD*

$(x)=$ elemento encontrado en la normativa autonómica 